\title{
Autosomal recessive lipid storage myopathy (probable carnitine deficiency)
}

\author{
C. ALMOG, K. FRIED, R. REIF, J. ZIEGHELBOIM, AND G. LEWINSOHN
}

From the Departments of Medicine, Genetics, Pathology, and Intensive Care, Asaf Harofe Hospital, Tel-Aviv University Medical School, Zerifin, Israel

SUMMARY Two sisters died at the age of 17 and 19, respectively, of a myopathy with exacerbations and remissions characterised by pain and weakness of muscles which ended fatally with lactic acidosis and respiratory failure. The clinical picture was very similar to that described in some cases of carnitine deficiency and the histochemical finding of many lipid-filled vacuoles in muscle fibres and the electron microscopical findings were identical to those reported in that disease. The finding of affected sisters supports autosomal recessive mode of inheritance.

Bradley et al. (1969) described a myopathy associated with abnormal lipid metabolism in skeletal muscle. Engel and Angelini (1973) and Engel et al. (1974) defined carnitine deficiency of human skeletal muscle with associated lipid storage myopathy. Since then further cases of lipid storage myopathy with carnitine deficiency have been described (Markesbery et al., 1974; Karpati et al., 1975; Smyth et al., 1975; Vandyke et al., 1975; Angelini et al., 1976; Boudin et al., 1976; Isaacs et al., 1976; Cornelio et al., 1977; Engel et al., 1977).

The purpose of the present report is to describe two sisters who died having presented a typical clinical picture of the disease. The genetics, age of onset, and age at death will be discussed.

\section{Family report}

The proband (case 1) and case 2 (Fig. 1) were the two oldest sisters of a sibship of eight. The parents were both Moroccan Jews but were not known to be

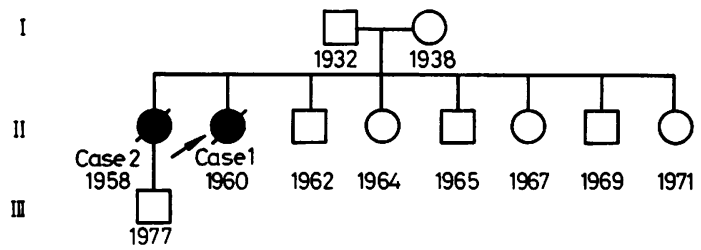

Fig. 1 Family pedigree. All family members except the infant of case 2 were examined. Year of birth given under each symbol.

Received for publication 16 February 1979 related. There were no known cases of muscle disease in the family. The parents and all sibs were examined and their age in years in 1978 is given in brackets: father (46), mother (40), brother (16), sister (14), brother (13), sister (11), brother (9), and sister (7). They all had normal serum CK levels and no pathological findings on physical examination. The only son of case 2 was reported to be in good health at the age of 19 months.

\section{Case 1}

\section{CLINICAL FINDINGS}

The proband, the younger sister of case 2, was born in June 1960 . Her early development was normal. She was first admitted to hospital because of febrile convulsions when she was 2 years old and again at the age of 3 years. In her third year she had hypochromic anaemia, upper respiratory infections, and an episode of acute gastroenteritis with hepatosplenomegaly. At the age of 4 years she was admitted to hospital with clouded consciousness, slightly stiff neck, and a history of fever and vomiting of 6 days and macuilopapular eruption of 2 days. There were signs of bronchopneumonia on $x$-ray examination. The diagnosis of morbilli, encephalitis postmorbilli, and bronchopneumonia postmorbilli was recorded. During her hospital stay of over 2 weeks 'ataxia' was noticed which decreased towards her discharge.

The patient had had painful and swollen leg muscles as well as muscle weakness since she was 12 years old. She noticed fluctuation in her disability. At age 15: years she was admitted to hospital because of sudden increase of weakness in the lower limbs. 
Physical examination showed decreased muscle power with slight wasting, especially in the thighs. The gastrocnemius muscles were pseudohypertrophic and tender. CK level reached $8150 \mathrm{IU} / \mathrm{l}, \mathrm{LDH} 1800$ IU/1, SGOT $1720 \mathrm{IU} / 1$. Electromyography was pathological but did not give conclusive results. Muscle biopsy from the gastrocnemius was interpreted as necrotising myopathy. Treatment was started with $50 \mathrm{mg}$ prednisolone daily and the patient was maintained on a $20 \mathrm{mg}$ daily dose. Her condition improved and she was able to return home but there was marked fluctuation in her clinical condition and she needed a further admission to hospital at the age of 16 years and 2 months. Her final admission to hospital was at the age of 17 years and 2 months; her condition deteriorated and her muscle pain and weakness increased. Three weeks after her admission she developed respiratory arrest and later cardiac arrest. She was successfully resuscitated and returned to consciousness. She developed hyperglycaemia that was corrected. A severe metabolic acidosis developed that did not respond to treatment and the patient went into acute renal failure and died from pulmonary oedema.

\section{PATHOLOGY}

At necropsy the gross pathological findings included marked pulmonary oedema, atelectasis of both lower lobes, as well as acute congestion of liver, spleen, brain, and kidneys. The histological examination of different skeletal muscles showed diffuse pathological changes. The involved muscle fibres may correspond to type I fibres. The affected muscle fibres were sometimes enlarged, sometimes atrophied with an eosinophilic sarcoplasm and often internal nuclei. The cross striations could sometimes still be recog-

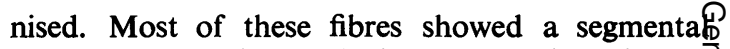
necrosis, some with marked phagocytosis and som with vacuolisation. The muscle with the most severe changes showed a definite increase in en? domysial fibrous tissue. A fat stain showed the abnormal fibres to contain a large amount of sudanophilic material in small droplets throughou的. the cytoplasm (Fig. 2). These histological change $\$$ were most marked in the gastrocnemius musclea Somewhat less severe were the changes in the femoralis, and the psoas, diaphragm, and the inter? costal muscles also showed a moderate involvement. Histological examination of the liver showed fine fa. droplets in a relatively large number of hepatocytes:The heart showed, in addition to lipofuscin, a fes fine fat droplets in some fibres. Early bronchoo pneumonia was seen. The findings are compatible with lipid storage myopathy with carnitine deficiencyê

\section{Case 2}

\section{CLINICAL FINDINGS}

The oldest sister was born in December 1958. Sh@ developed normally. At the age of 12 years she was admitted to hospital after falling from a height of क metres; a fracture of the skull in the right orbitat area was diagnosed on $x$-ray examination. The liges was not palpable, and recovery was unevent From the age of 15 she noticed weakness on faos longed physical exercise and therefore she stoppe participating in trips that included long walks. She noticed fluctuation in her disability. At the age of $17 \frac{1}{2}$ years she was admitted to hospital for observa? tion after the proband (case 1) was admitted to hospital because of suspected myopathy. Complete physical and neurological examination did not show

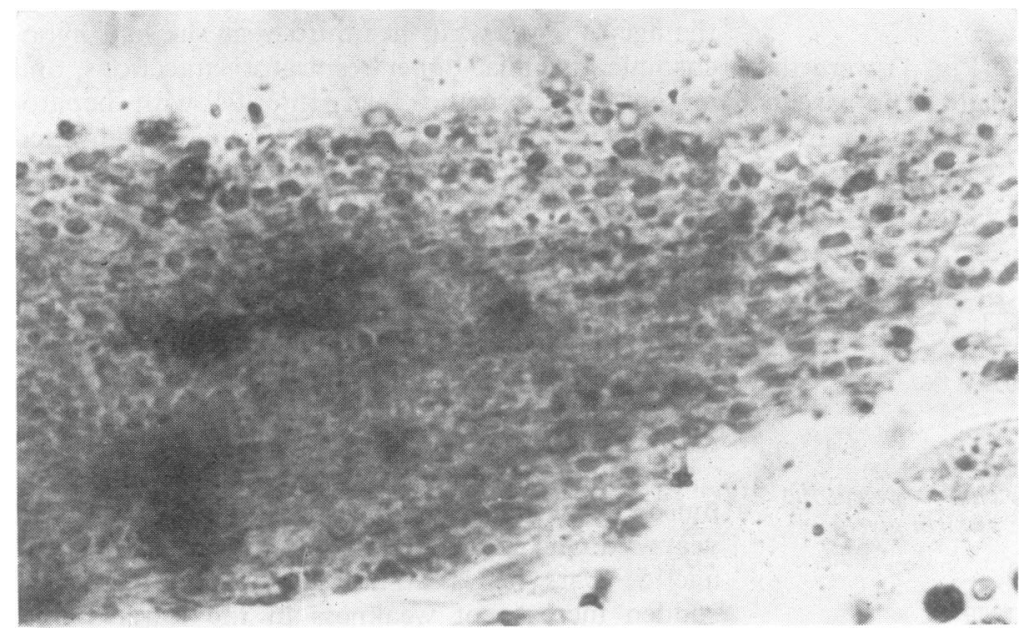

Fig. 2 Case 1, skeletal muscle $\frac{\mathrm{D}}{\mathrm{O}}$ shows a large number of fat dropo lets within the cytoplasm. (Sudar red. $\times 800$, original magnification 
any pathological finding. Electromyography showed only reduced interference pattern on maximal exertion. Chest $x$-ray examination was normal. Serum CK level was $102 \mathrm{IU} / 1$.

The patient married and became pregnant. Her weakness increased and she became bedridden in the sixth month of pregnancy. Admission to hospital at that time revealed extreme weakness. She was unable to walk or lift her arms above her head. She could not open her mouth to the full extent. CK level reached $7568 \mathrm{IU} / 1$, LDH $6380 \mathrm{IU} / \mathrm{l}$, SGOT 1100 IU/l, SGPT 366 IU/1, aldolase 240 IU/l. Treatment with $60 \mathrm{mg}$ prednisolone daily was started. Her condition improved within a week and the enzyme levels tended to decrease towards the normal range. She left hospital in a satisfactory condition 4 weeks after admission with a maintenance dose of $5 \mathrm{mg}$ prednisolone daily.

Two months later at the age of $18 \frac{1}{2}$ years she gave birth to a healthy son in an uneventful delivery, and she stopped taking steroids. Two months after delivery she had an episode of muscle pain and weakness and she experienced difficulty in walking on the tip of her toes. CK and LDH in serum were again markedly raised. Four months later when she was 19 years old her serum CK level returned to normal. A biopsy from the gastrocnemius showed myopathic changes with many very small fat droplets in the muscle fibres. Electron microscopy (Fig. 3) showed vacuoles of varying size in muscle fibres with some vacuoles coalescing and forming what appeared to be empty spaces. At the age of 19 years and 3 months she was admitted to hospital for the last time because of difficulty in swallowing and extreme muscle weakness. Again serum CK and LDH levels were markedly increased. Because of difficulty in use of the masseters and in tongue movements she needed gastric tube feeding. Severe lactic acidosis

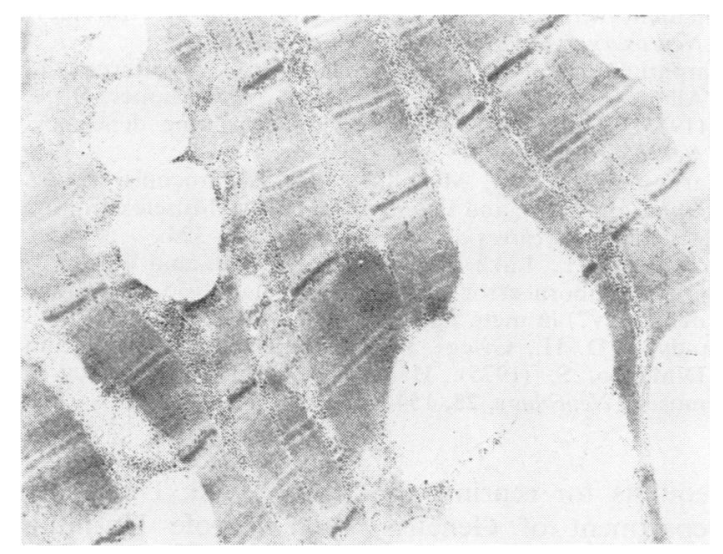

Fig. 3 Case 2, muscle biopsy electron miscroscopy. and respiratory difficulties developed that needed tracheostomy and assisted respiration. The serum lactic acid level reached $9490 \mu \mathrm{mol} / 1$ (normal 500 $1300 \mu \mathrm{mol} / \mathrm{l}$ ). The lactic acidosis was corrected by large doses of bicarbonate. Myoglobinuria was noticed for the first time. Massive doses of steroids had no effect and her clinical condition deteriorated. She could not move her limbs and only slight movements of the fingers were possible. She died three weeks after her admission to hospital having developed pneumonia and most probably septic shock.

\section{PATHOLOGY}

Permission for full necropsy was not granted, but post mortem examination of a piece of muscle showed atrophy and segmental necrosis of muscle fibres. Some fibres were hypertrophic and the cytoplasm was vacuolated. There were signs of regeneration and increase of connective tissue among the fibres and between muscle bundles. Special fat staining showed many sudanophilic droplets in the cytoplasm (Fig. 4) confirming the diagnosis of lipid storage myopathy.

\section{Discussion}

The affected sisters described in the present report had a lipid storage myopathy with a clinical course and histochemical and electron microscopical findings characteristic of carnitine deficiency, but carnitine levels were not measured. Fewer than 20 cases of carnitine deficiency have been described and in no case were sibs reported. Cornelio et al. (1977), who reported three unrelated cases in north Italy, mentioned that a sister of their case 2 died at the age of 5 years of a condition clinically similar to that of the proband. Only the parents of the case reported by Bradley et al. $(1969,1972,1978)$ were first cousins. The present family supports an autosomal recessive mode of inheritance of carnitine deficiency. The relatively low frequency of consanguinity among the parents suggests that the disease is not very rare and that many cases have gone undiagnosed.

The age of onset is usually in childhood and only two cases had an onset after the age of 18. The case of Bradley et al. (1969) first showed signs at the age of 23, and the patient of Markesbery et al. (1974) at the age of 46. Those who have been reported after death have died either in childhood or as young adults. The patient of Boudin et al. (1976) is similar to our case 2 in that she died 10 months after she gave birth to a child.

At different times during the course of disease the two sisters were suspected of having muscular dystrophy, chronic polymyositis, and myasthenia gravis, but all these diseases were easily ruled out by the appropriate tests and by the fatal course of the 


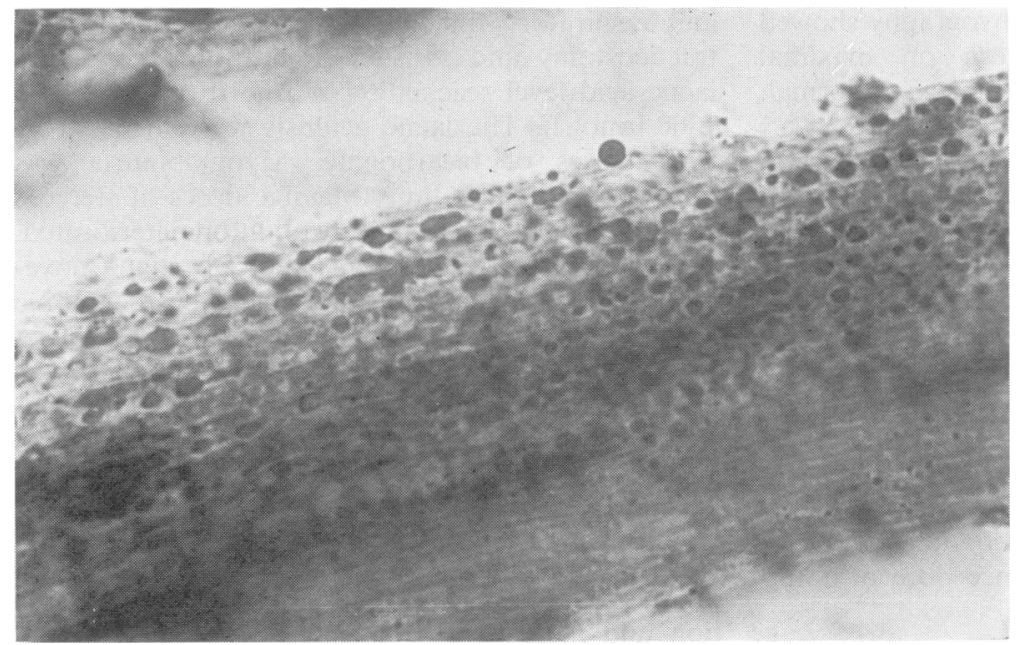

Fig. 4 Case 2, skeletal muscle shows multiple fat droplets within the cytoplasm. (Sudan red. $\times 800$, original magnification.)

disease, ending in lactic acidosis in case 2 and metabolic acidosis in case 1 . This was judged in retrospect to be also the result of increased lactic acid but was not tested at the time. Treatment with prednisolone as suggested by Engel and Siekert (1972), Engel and Angelini (1973), and Vandyke et al. (1975) seemed to benefit the patients for a period, but it is extremely difficult to evaluate the therapy in a disease that has an intermittent course and spontaneous remissions.

As the six sibs are younger, it cannot be ruled out that some of them may still develop the disease.

The sex ratio of the 13 unrelated previously reported cases was 6 males : 7 females, as would be expected if the disease had an autosomal recessive mode of inheritance. In most cases the number of sibs was not reported so that no segregation ratio can be calculated.

We are grateful to Professor U. Sandbank for the electron microscopy and to $\mathrm{Dr}$ E. Hegesh for examination of the blood lactic acid levels.

\section{References}

Angelini, C., Lucke, S., and Cantasutti, F. (1976). Carnitine deficiency of skeletal muscle. Report of a treated case. Neurology, 26, 633-637.

Boudin, G., Mikol, J., Guillard, A., and Engel, A. G. (1976). Fatal systemic carnitine deficiency with lipid storage in skeletal muscle, heart, liver and kidney. Journal of the Neurological Sciences, 30, 313-325.

Bradley, W. G., Hudgson, P., Gardner-Medwin, D., and Walton, J. N. (1969). Myopathy associated with abnormal lipid metabolism in skeletal muscle. Lancet, 1, 495-498.

Bradley, W. G., Jenkison, M., Park, D. C., Hudgson, P., Gardner-Medwin, D., Pennington, R. J. T., and Walton, J. N. (1972). A myopathy associated with lipid storage. Journal of the Neurological Sciences, 16, 137-154.

Bradley, W. G., Tomlinson, B. E., and Hardy, M. (1978). Further studies of mitochondrial and lipid storage myopathies. Journal of the Neurological Sciences, 35, 201-210.
Cornelio, F., Di Donato, S., Peluchetti, D., Bizzi, A.? Bertagnolio, B., D'Angelo, A., and Weismann, U. (1977) Fatal cases of lipid storage myopathy with carnitine deficiency. Journal of Neurology, Neurosurgery and Psy? chiatry, 40, 170-178.

Engel, A. G., and Angelini, C. (1973). Carnitine deficiency of human skeletal muscle with associated lipid storage myopathy: a new syndrome. Science, 179, 899-902.

Engel, A. G., Angelini, C., and Nelson, R. A. (1974). Ideptie fication of carnitine deficiency as a cause of human bido storage myopathy. In Exploratory Concepts. II. Confot Mechanisms in Development and Function of Musele pp. 601-617, ed A. T. Milhorat. Excerpta Med;ea, Amsterdam.

Engel, A. G., Banker, B. Q., and Eiben, R. M. (1977) Carnitine deficiency: clinical, morphological and biof chemical observation in a fatal case. Journal of Neurology? Neurosurgery and Psychiatry, 40, 313-322.

Engel, A. G., and Siekert, R. G. (1972). Lipid storage myopathy responsive to prednisone. Archives of Neurology 27, 174-181.

Isaacs, H., Heffron, J. J. T., Badenhorst, M., and Pickering. A. (1976). Weakness associated with the pathologicat presence of lipid in skeletal muscle: a detailed study of patient with carnitine deficiency. Journal of Neurology Neurosurgery and Psychiatry, 39, 1114-1123.

Karpati, G., Carpenter, S., Engel, A. G., Watterns, G Allen, G., Rothman, S., Klassen, G., and Mamer, O. AO (1975). The syndrome of systemic carnitine deficiency?

Neurology, 25, 16-24.
Markesbery, W. R., McQuillen, M. P., Procopis, P. G.? Harrison, A. R., and Engel, A. G. (1974). Muscle carnitine deficiency. Archives of Neurology, 31, 320-324.

Smyth, D. P. L., Lake, B. D., MacDermot, J., and Wilson, hy (1975). Inborn error of carnitine metabolism ("carniting deficiency") in man. Lancet, 1, 1198-1199.

Vandyke, D. H., Griggs, R. C., Markesbery, W. R., an\$ DiMauro, S. (1975). Hereditary carnitine deficiency of muscle. Neurology, 25, 154-159.

Requests for reprints to Dr K. Fried, Universit Department of Genetics, Asaf Harofe Hospital Zerifin, Israel. 